\title{
Stereotactic ablative body radiotherapy boost for cervical cancer when brachytherapy boost is not feasible
}

\author{
Tae Hoon Lee ${ }^{1}$, Changhoon Song ${ }^{2}$, In Ah Kim², Jae-Sung Kim², Yong Beom Kim³ ${ }^{3}$ Kidong Kim³ , Jae Hong No ${ }^{3}$, \\ Dong Hoon Suh ${ }^{3}$, Jin-Beom Chung ${ }^{2}$ and Keun-Yong Eom ${ }^{2 *}$ (]
}

\begin{abstract}
Background: The purpose of this study was to analyze the treatment efficacy and safety of stereotactic ablative body radiotherapy (SABR) boost for cervical cancer patients not amenable to brachytherapy.

Methods: A retrospective review of the medical records from single institution of 25 eligible patients was performed. The patients underwent pelvic radiotherapy (RT) in 25 or 28 fractions with a median dose of 45 Gy (range 44-50.4 Gy). SABR boost was delivered after pelvic RT, with a median dose of 25 Gy (range 20-33 Gy), and a median fraction number of 5 (range 4-6). 21 patients with a follow-up period of more than one year were included in the toxicity analysis, and hematuria and hematochezia that occurred later than 3 months after the RT were graded.

Results: The median follow-up period after radiotherapy was 2.85 years (range $0.33-6.60$ ). The 3-year local control, locoregional control, disease-free survival, and overall survival rates were $80.9 \%, 75.8 \%, 40.9 \%$, and $77.1 \%$, respectively. 5 patients experienced grade 3 toxicity ( 3 genitourinary, 3 gastrointestinal), and no grade $4-5$ toxicity was reported. Univariate analysis showed that cumulative $D_{2 c c}$ in equivalent dose in $2 \mathrm{~Gy}$ fractions (EQD2) of rectum was marginally predictive for any grade of hematochezia $(P=0.051)$. Cumulative $D_{2 c c} E Q D 2$ of bladder was not predictive for hematuria. In the receiver operating characteristic (ROC) curve analysis, the optimal threshold of cumulative rectal $D_{2 c c} E Q D 2$ was 81.2 Gy for any grade of hematochezia.
\end{abstract}

Conclusion: SABR boost for cervical cancer was effective and tolerable. Although it cannot substitute brachytherapy, it can be a treatment option when brachytherapy is not possible.

Keywords: Cervical cancer, Stereotactic body radiotherapy, Hematuria, Hematochezia

\section{Introduction}

Patients with locally advanced cervical cancer are usually treated with concurrent chemoradiation and brachytherapy boost. It is well known that brachytherapy is an essential component of treatment for locally advanced cervical cancer, and some reports have shown that brachytherapy

\footnotetext{
${ }^{*}$ Correspondence: 978sarang@hanmail.net

2 Department of Radiation Oncology, Seoul National University Bundang

Hospital, 82 Gumi-ro 173 beon-gil, Bundang-gu, Seongnam 13620, Republic of Korea

Full list of author information is available at the end of the article
}

is associated with better treatment outcomes, including cancer-specific survival [1]. Despite the importance of brachytherapy, the availability of brachytherapy is relatively limited because it is operator dependent, and failure to maintain the quality of brachytherapy can reduce the treatment efficacy [2]. In addition, a small proportion of patients cannot undergo brachytherapy due to an obstructing tumor mass, anatomical variations, or medical comorbidities. For patients who are unable to undergo brachytherapy boost due to the aforementioned reasons, external beam radiotherapy (EBRT) boost can original author(s) and the source, provide a link to the Creative Commons licence, and indicate if changes were made. The images or other third party material in this article are included in the article's Creative Commons licence, unless indicated otherwise in a credit line to the material. If material is not included in the article's Creative Commons licence and your intended use is not permitted by statutory regulation or exceeds the permitted use, you will need to obtain permission directly from the copyright holder. To view a copy of this licence, visit http://creativecommons.org/licenses/by/4.0/. The Creative Commons Public Domain Dedication waiver (http://creativeco mmons.org/publicdomain/zero/1.0/) applies to the data made available in this article, unless otherwise stated in a credit line to the data. 
be an alternative. Some studies with small sample sizes evaluated EBRT boost, and reported that the outcomes were sufficiently acceptable to consider EBRT boost when brachytherapy is not available [3, 4].

Recently, stereotactic ablative body radiotherapy (SABR) has been applied for the treatment of several tumors arising from various organs. Compared with cervical EBRT boost reported in earlier studies, a higher biologically equivalent dose can be delivered by the SABR technique. Additionally, as SABR can deliver a higher dose per fraction, the proposed dose-fractionation schemes for cervical SABR boost are more similar to the schemes for brachytherapy when comparing with those for EBRT boost with conventional fractionation [5], although central dose would still be limited. As a result, cervical SABR boost is a promising option for cervical cancer which is not amenable to brachytherapy boost; nevertheless, the feasibility and safety of SABR boost should be evaluated. The purpose of this study was to analyze the treatment efficacy and toxicity of SABR boost in cervical cancer patients.

\section{Methods \\ Study population}

The medical records of patients from single institution with histologically confirmed cervical cancer who underwent curative radiotherapy (RT) with SABR boost between 2013 and 2019 were retrospectively reviewed, and 25 patients were found to be eligible. These patients were not suitable for brachytherapy boost and had no prior pelvic RT. Among them, 20 (80.0\%) patients were unable to undergo brachytherapy due to failure of intracavitary device insertion because of a narrow vagina or cervical os fibrosis. These patients found to be ineligible to undergo brachytherapy after cervical os cannulation effort by an experienced gynecologist. Two (8.0\%) patients had large tumors that could not be adequately irradiated by brachytherapy. One patient had extreme obesity, making the evaluation of the cervix and uterine canal impossible. Among the remaining two patients, one patient experienced intolerable pain with an intracavitary device, while the other patient was unable to position appropriately due to left spastic hemiplegia.

\section{Pelvic radiotherapy and chemotherapy}

All patients underwent whole pelvic RT. For all but one patient, the radiation dose to the pelvis was $45-50.4 \mathrm{~Gy}$, and the dose per fraction was $1.8 \mathrm{~Gy}$. One patient had a pelvic dose of $44 \mathrm{~Gy}$, and the dose per fraction was 2 Gy. Both 3-dimensional conformal radiation therapy (3D-CRT) and intensity-modulated radiation therapy (IMRT) techniques were used, but IMRT was applied to only two (8.0\%) patients. For all cases, the clinical target volume (CTV) was delineated based on previously published consensus guidelines [6]. As stated in the guidelines, the cervical CTV included all gross tumors, cervix, uterus, parametria, and upper vagina, while the nodal CTV included the common iliac, internal iliac, external iliac, obturator, and presacral lymph nodes. If suspected metastatic lymph nodes in the common iliac lymph node area or above were detected by staging examinations, para-aortic lymph nodes were added to the nodal CTV. The pelvic planning target volume (PTV) was calculated by adding a $5 \mathrm{~mm}$ margin around the cervical and nodal CTV. For the 3D-CRT technique, "box field" consisting of parallel opposed AP-PA fields and two lateral opposed fields with $15 \mathrm{MV}$ photon beams was set up to cover the pelvic PTV. For IMRT, the volumetricmodulated arc therapy (VMAT) technique was applied, and two 360-degree arcs of beams with a $10 \mathrm{MV}$ photon beam were used. Boost to gross lymph nodes was added in patients with suspected metastatic lymph nodes. The lymph node boost was delivered sequentially in eight (32.0\%) patients, and concurrently in two $(8.0 \%)$ patients using IMRT plans by a simultaneous integrated boost technique. The radiation dose for the lymph node boost was variable (range 5.4-14.4 Gy). For consistent bladder filling, the patients were instructed to restrain from voiding urine for one hour after the last voiding and drink two cups of water.

During pelvic RT, concurrent cisplatin was administered to medically fit patients. A weekly cisplatin regimen was applied to $12(48.0 \%)$ patients, and a tri-weekly cisplatin regimen was applied to five $(20.0 \%)$ patients. For patients underwent weekly cisplatin regimen, the median number of chemotherapy cycle was 6 (range 4-7). Among these patients, 10 patients were able to complete planned chemotherapy. For patients underwent tri-weekly cisplatin regimen, the median number of chemotherapy cycle was 3 (range 2-3), and 4 patients were able to complete planned chemotherapy. Four patients underwent consolidation chemotherapy after concurrent chemoradiation. In three patients, the consolidation regimen consisted of paclitaxel and cisplatin with or without bevacizumab. One patient underwent a cycle of consolidation triweekly cisplatin after concurrent chemoradiation.

\section{SABR boost and follow-up}

After completing pelvic RT, the patients underwent SABR boost to the cervix. The patients underwent a simulation computed tomography (CT) scan for SABR boost 1 week before the end of pelvic RT. To reduce spatial variability from the adjacent organs, a Foley catheter was inserted and 150-200 cc of normal saline was infused into the bladder to maintain a reproducible bladder volume, along with emptying of the rectum by an enema. 
These procedures were performed before the simulation CT scan and repeated for every fraction. Pelvic magnetic resonance imaging (MRI) was performed in the same day with RT simulation, and the MRI image was fused with the simulation CT scan.

Regarding the dose-fractionation scheme and target volume delineation, an institutional protocol was implemented in late 2014. Before this, dose-fractionation was variable (range, total 20-33 Gy, 4-6 fractions), and the exact procedure to delineate the target volume was inconsistent. Fifteen patients were treated based on the institutional protocol. Dose-fractionation was 25 Gy in 5 fractions for these patients, except for two patients who received 20 Gy in 4 fractions due to unacceptable organ at risk (OAR) dose. The principles of target volume delineation in the protocol were as follows. The gross tumor volume (GTV) included residual tumor in pre-SABR image. The clinical target volume for the $25 \mathrm{~Gy}$ area $\left(\mathrm{CTV}_{25 \mathrm{~Gy}}\right)$ was GTV plus the entire cervix. $\mathrm{CTV}_{25 \mathrm{~Gy}}$ was expanded by $5 \mathrm{~mm}$ in all directions to make the planning target volume for the $25 \mathrm{~Gy}$ area $\left(\mathrm{PTV}_{25 \mathrm{~Gy}}\right)$. $\mathrm{CTV}_{20 \mathrm{~Gy}}$ was defined as $\mathrm{CTV}_{25 \mathrm{~Gy}}$ with a $10 \mathrm{~mm}$ margin expansion in the superior and inferior directions. $\mathrm{PTV}_{20 \mathrm{~Gy}}$ was constructed by $5 \mathrm{~mm}$ expansion of $\mathrm{CTV}_{20 \mathrm{~Gy}}$. Rectal V25 of SABR was limited below 25\%. For planning SABR, the VMAT technique with two 360-degree arcs with a $10 \mathrm{MV}$ photon beam was applied, except for one patient who was treated with a static IMRT plan. An example of SABR plan was illustrated in Fig. 1.

The patients were followed up with clinical examination, tumor marker test and radiologic examination at 1 month after RT. Thereafter, patients were recommended to follow-up by clinical examination and tumor marker test with 3-month interval and radiologic examination with 3 - to 6-month interval for 2 years. The follow-up interval was doubled for the third to fifth years. Tumor marker test included SCC, CA-125, and CEA if the tumor pathology was adenocarcinoma. Radiological examination included CT, MRI, or positron emission tomography (PET)/CT scan.

\section{Clinical outcomes and toxicity profile}

The clinical outcomes in this study were local control (LC), locoregional control (LRC), progression-free survival (PFS), and overall survival (OS), which were calculated using the Kaplan-Meier method. These treatment outcomes were measured from the date of the end of RT for each defined event. An LC event was defined as

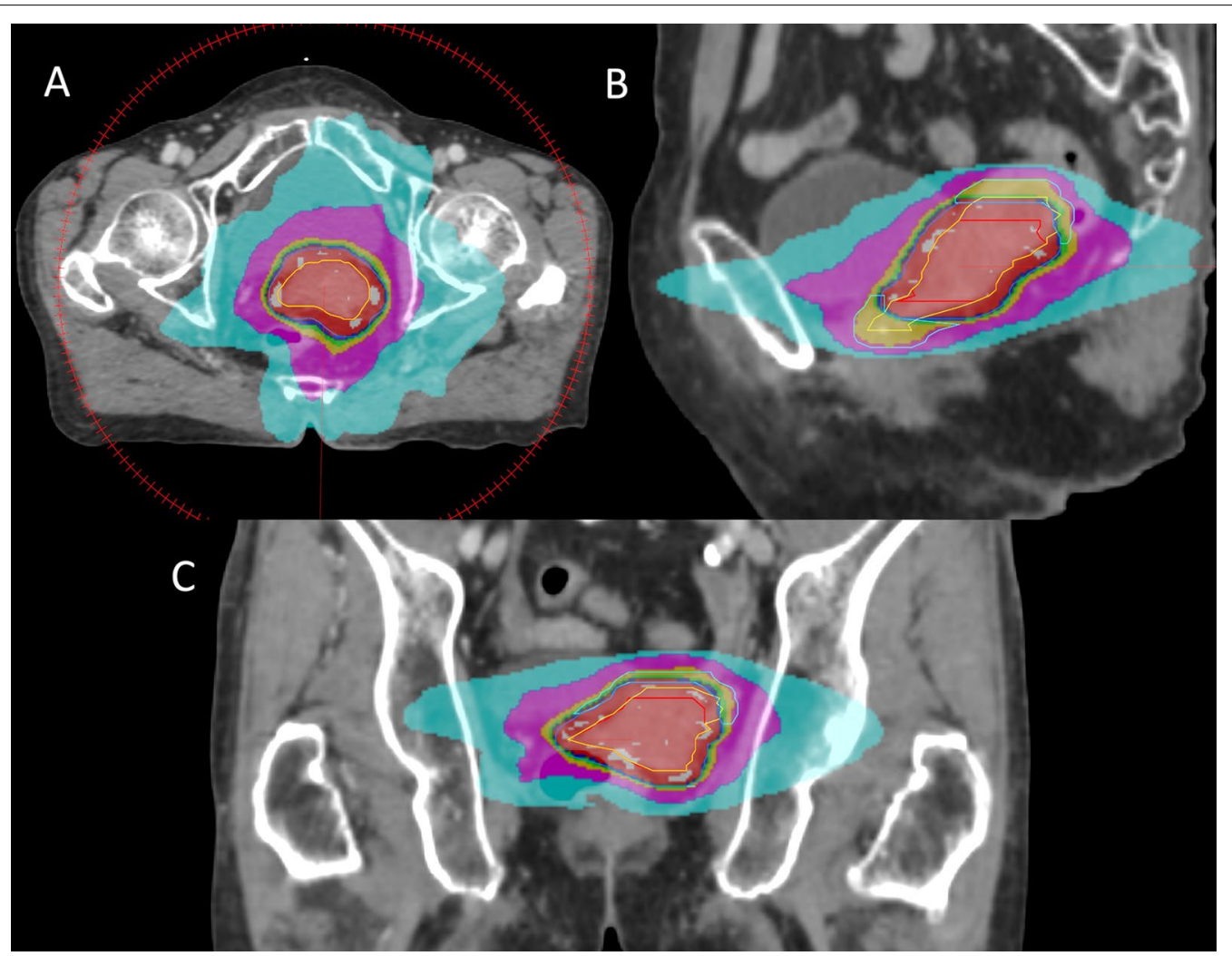

Fig. 1 An example of cervical SABR boost plan. (a) Axial, (b) sagittal, and (c) coronal cut of representative SABR plan. Red, yellow, blue and cyan lines indicate $\mathrm{CTV}_{25 \mathrm{G}^{\prime}} \mathrm{CTV}_{20 \mathrm{~Gy}} \mathrm{PTV}_{25 \mathrm{~Gy}}$ and PTV $20 \mathrm{~Gy}$, respectively 
a recurrence in the treated cervix or parametrium, while an LRC event was defined as a recurrence in the pelvic area. For OS, the event was defined as the death of the patient, whereas for PFS, the event was defined as disease progression or death.

For analysis of RT toxicity, medical records of the patients with a follow-up period of more than one year were reviewed, and 21 patients were included. New occurrences or worsening of the toxicities more than 3 months after the completion of RT were recorded and graded by the Common Terminology Criteria for Adverse Events (CTCAE) version 5. Most reported adverse events were genitourinary and gastrointestinal toxicities. Hematuria and hematochezia were also relatively well-documented throughout the medical records. Therefore, we classified the toxicities into five categories: urinary symptoms (other than hematuria), hematuria, proctitis (other than hematochezia), hematochezia, and others. Additionally, the rate of freedom from toxicity was calculated for hematuria and hematochezia using the Kaplan-Meier method. For dose-response analysis, cumulative dose delivered to $2 \mathrm{cc}$ volume $\left(\mathrm{D}_{2 \mathrm{cc}}\right)$ of the bladder and rectum was used. Cumulative $D_{2 c c}$ was calculated by adding equivalent dose in 2 Gy fractions (EQD2) with $\alpha / \beta$ of 3 of prescribed dose-fractionation of pelvic RT to EQD2 of $\mathrm{D}_{2 \mathrm{cc}}$ of bladder and rectum from the SABR plan. Univariate analysis with logistic regression was performed to find whether the cumulative $D_{2 c c}$ of rectum and bladder was predictive for occurrence of hematochezia and hematuria. Receiver operating characteristic (ROC) curves were created for the toxicities with cumulative $\mathrm{D}_{2 \mathrm{cc}}$ of these OARs to determine the threshold dose when the univariate analysis showed statistical significance. The optimal threshold of cumulative $D_{2 c c}$ was calculated using the Youden index method. All statistical analyses were performed using R 3.6.0 (The R Foundation for Statistical Computing, Vienna, Austria).

\section{Results}

\section{Patient characteristics and radiotherapy specifics}

The characteristics of all patients included in this study are summarized in Table 1. The median follow-up period for all patients was 2.85 years (range $0.33-6.60$ years). The median age at diagnosis was 73.7 years, and the average was 67.7 years (range, 29.6 to 88.2 years). Most patients (80.0\%) had good performance status ranging from 0 to 1 using the Eastern Cooperative Oncology Group (ECOG) performance status scale, although some (20.0\%) patients with poor performance status were also included. More than half of the patients (56.0\%) had cervical cancer with International Federation of Gynecology and Obstetrics (FIGO) stage III or IV. Two patients had distant metastasis at the start of RT. One patient had supraclavicular
Table 1 Patient characteristics

\begin{tabular}{|c|c|}
\hline Characteristics & Number \\
\hline Total & $25(100.0 \%)$ \\
\hline Age (years, median) & 73.7 (range 29.6-88.2) \\
\hline \multicolumn{2}{|l|}{ ECOG performance status } \\
\hline 0 & $1(4.0 \%)$ \\
\hline 1 & $19(76.0 \%)$ \\
\hline 2 & $4(16.0 \%)$ \\
\hline 3 & $1(4.0 \%)$ \\
\hline \multicolumn{2}{|l|}{ FIGO stage (2018) } \\
\hline । & $1(4.0 \%)$ \\
\hline$\|$ & $10(40.0 \%)$ \\
\hline III & $9(36.0 \%)$ \\
\hline IV & $5(20.0 \%)$ \\
\hline \multicolumn{2}{|l|}{ Pathology } \\
\hline Squamous cell carcinoma & $20(80.0 \%)$ \\
\hline Adenocarcnoma & $3(12.0 \%)$ \\
\hline Poorly differentiated carcinoma & $2(8.0 \%)$ \\
\hline \multicolumn{2}{|l|}{ Pelvic organ invasion } \\
\hline Yes & $4(16.0 \%)$ \\
\hline No & $21(84.0 \%)$ \\
\hline \multicolumn{2}{|l|}{ Pelvic lymph node metastasis } \\
\hline Yes & $9(36.0 \%)$ \\
\hline No & $16(64.0 \%)$ \\
\hline \multicolumn{2}{|l|}{ Paraaortic lymph node metastasis } \\
\hline Yes & $6(24.0 \%)$ \\
\hline No & $19(76.0 \%)$ \\
\hline \multicolumn{2}{|l|}{ Distant metastasis } \\
\hline Yes & $2(8.0 \%)$ \\
\hline No & $23(92.0 \%)$ \\
\hline \multicolumn{2}{|l|}{ Concurrent chemotherapy } \\
\hline Yes & $17(68.0 \%)$ \\
\hline No & $8(32.0 \%)$ \\
\hline \multicolumn{2}{|l|}{ Consolidation chemotherapy } \\
\hline Yes & $4(16.0 \%)$ \\
\hline No & 21 (84.0\%) \\
\hline
\end{tabular}

ECOG, eastern cooperative oncology group; FIGO, International Federation of Gynecology and Obstetrics

lymph node metastasis, and supraclavicular lymph node was also included in the RT field. Another patient had a questionable metastatic lesion in the liver in initial MRI. Re-evaluation of the suspected hepatic lesion after concurrent chemoradiation was planned, and the lesion progressed 3 months after the completion of concurrent chemoradiation. Additional chemotherapy was not administered due to refusal of the further treatment.

The specifics of pelvic RT and SABR boost are summarized in Table 2. The median dose and fractionation for pelvic RT was 45 Gy in 25 fractions. For the SABR boost, the median dose and fractionation were $25 \mathrm{~Gy}$ and 
Table 2 Radiotherapy specifics

\begin{tabular}{|c|c|}
\hline \multicolumn{2}{|l|}{ Pelvic RT technique } \\
\hline 3D-CRT & $23(92.0 \%)$ \\
\hline IMRT & $2(8.0 \%)$ \\
\hline Pelvic RT dose (Gy, median) & 45 (range 44-50.4) \\
\hline Pelvic RT fractionations (median) & 25 (range 22-28) \\
\hline Pelvic RT BED (Gy, median, $\alpha / \beta=3$ ) & 72 (range $72-80.64$ ) \\
\hline Pelvic RT BED (Gy, median, $a / \beta=10)$ & 53.1 (range 52.8-59.47) \\
\hline \multicolumn{2}{|l|}{ Lymph node boost } \\
\hline Sequential & $8(32.0 \%)$ \\
\hline Simultaneous & $2(8.0 \%)$ \\
\hline No & $15(60.0 \%)$ \\
\hline \multicolumn{2}{|l|}{ Bladder (pelvic RT) } \\
\hline Mean dose (Gy, average \pm SD) & $46.3 \pm 3.8$ \\
\hline Mean BED (Gy, average $\pm S D, a / \beta=3)$ & $73.9 \pm 7.3$ \\
\hline Maximum dose (Gy, average \pm SD) & $50.0 \pm 3.4$ \\
\hline Maximum BED (Gy, average $\pm S D, a / \beta=3$ ) & $82.2 \pm 6.9$ \\
\hline \multicolumn{2}{|l|}{ Rectum (pelvic RT) } \\
\hline Mean dose (Gy, average $\pm \mathrm{SD}$ ) & $44.2 \pm 3.9$ \\
\hline Mean BED (Gy, average $\pm S D, a / \beta=3$ ) & $69.4 \pm 7.7$ \\
\hline Maximum dose (Gy, average \pm SD) & $49.4 \pm 2.5$ \\
\hline Maximum BED (Gy, average $\pm S D, a / \beta=3)$ & $80.9 \pm 4.6$ \\
\hline SABR boost dose (Gy, median) & 25 (range 20-33) \\
\hline \multicolumn{2}{|l|}{ SABR boost fractions } \\
\hline 4 & $4(16.0 \%)$ \\
\hline 5 & $17(68.0 \%)$ \\
\hline 6 & $4(16.0 \%)$ \\
\hline SABR boost BED (Gy, median, $a / \beta=3$ ) & 66.7 (range 53.3-93.5) \\
\hline SABR boost BED ( $G y$, median, $a / \beta=10)$ & 37.5 (range $30-51.2$ ) \\
\hline Total volume of PTV (cc) & 120.0 (range $70.9-412.1$ ) \\
\hline \multicolumn{2}{|l|}{ Bladder (SABR) } \\
\hline Mean dose (Gy, average \pm SD) & $12.3 \pm 4.4$ \\
\hline Mean BED (Gy, average $\pm S D, a / \beta=3$ ) & $23.1 \pm 11.6$ \\
\hline Maximum dose (Gy, average \pm SD) & $25.3 \pm 3.1$ \\
\hline Maximum BED (Gy, average $\pm S D, a / \beta=3)$ & $68.4 \pm 11.0$ \\
\hline $\mathrm{D}_{2 c c} \mathrm{EQD2}(\mathrm{Gy}$, average $\pm S D, a / \beta=3)$ & $31.4 \pm 6.8$ \\
\hline $\begin{array}{l}\text { Cumulative } D_{2 c c} E Q D 2 \text { (Gy, average } \pm S D \text {, } \\
\qquad / \beta=3\end{array}$ & $79.9 \pm 6.1$ \\
\hline \multicolumn{2}{|l|}{ Rectum (SABR) } \\
\hline Mean dose (Gy, average \pm SD) & $11.4 \pm 2.9$ \\
\hline Mean BED (Gy, average $\pm S D, a / \beta=3$ ) & $20.6 \pm 7.5$ \\
\hline Maximum dose (Gy, average \pm SD) & $24.5 \pm 3.5$ \\
\hline Maximum BED (Gy, average $\pm S D, a / \beta=3)$ & $65.2 \pm 12.7$ \\
\hline$D_{2 c c} E Q D 2(G y$, average $\pm S D, a / \beta=3)$ & $34.8 \pm 6.9$ \\
\hline $\begin{array}{l}\text { Cumulative } D_{2 c c} E Q D 2 \text { (Gy, average } \pm S D \text {, } \\
\quad \alpha / \beta=3\end{array}$ & $76.5 \pm 6.2$ \\
\hline
\end{tabular}

$\mathrm{RT}$, radiotherapy; 3D-CRT, three dimensional conformal radiotherapy; IMRT, Intensity-modulated radiation therapy; BED, biological equivalent dose; $\mathrm{SABR}$, stereotactic ablative body radiotherapy; SD, standard deviation; $\mathrm{D}_{2 c c^{\prime}}$ dose delivered to 2 cc volume; PTV, planning target volune
5 fractions, respectively, according to the institutional protocol. For simple summation of biologically effective dose (BED) with $\alpha / \beta$ of 10 from the prescribed dose fractionation scheme of pelvic RT and SABR boost, the median was 90.6 Gy (range 83.1-110.6 Gy). The median EQD2 was 75.5 Gy (range 69.3-92.2 Gy), which is lower than the recommended EQD2 for adding brachytherapy after EBRT [7]. Median total treatment time was 52 days (range 35-68 days). 4 patients had total treatment time more than 8 weeks (56 days).

\section{Treatment outcomes}

The 1-, 3-, and 5-year LC rates were $86.3 \%, 80.9 \%$, and $80.9 \%$, respectively, and the 1-, 3-, and 5-year LRC rates were $86.3 \%, 75.8 \%$, and $75.8 \%$, respectively. The OS rates at 1,3 , and 5 years were $91.7 \%, 77.1 \%$ and $69.4 \%$, respectively, while the PFS rates at 1,3 , and 5 years were $58.5 \%, 40.9 \%$, and $40.9 \%$, respectively. The most frequent failure pattern was distant failure, which occurred in eight $(32.0 \%)$ patients. Four $(16.0 \%)$ patients developed para-aortic lymph node metastasis after treatment. Four (16.0\%) patients experienced local failure, and two (8.0\%) patients had regional failure. No disease progression within the follow-up period was reported among 14 (56.0\%) patients. The treatment outcomes of all patients are presented in Fig. 2.

\section{Toxicity profile}

Toxicities that occurred later than 3 months after the end of RT are summarized in Table 3. All reported toxicities were either genitourinary or gastrointestinal, with the exception of grade 2 lower extremity edema. Five (23.8\%) patients had grade 3 adverse events. Three (14.3\%) patients experienced grade 3 hematuria, and three (14.3\%) patients experienced grade 3 hematochezia. There was no grade 4 or 5 toxicity. The median period for onset of hematuria was 23.9 months (range 7.7-50.4 months), and the median period for onset of hematochezia was 16.2 months (range 6.9-44.9 months). Among four patients with local failure, two grade 2 urinary symptoms other than hematuria, one grade 2 hematuria, one grade 1 proctitis other than hematochezia, one grade 1 hematochezia, and one grade 2 hematochezia were reported. The rates of freedom from hematuria and hematochezia are illustrated in Fig. 3. The 1-, 3-, and 5-year rates of freedom from any grade of hematuria were $95.2 \%, 58.5 \%$, and $46.8 \%$, respectively, while the 1 - and 3-year rates of freedom from any grade of hematochezia were $76.2 \%$ and $29.6 \%$, respectively. The $1-, 3-$, and 5-year rates of freedom from hematuria with grade 2 or worse were $95.2 \%, 73.2 \%$, and $61.0 \%$, respectively, while the 1- and 3-year rates for hematochezia with grade 2 or worse were $85.7 \%$ and $58.0 \%$, respectively. 

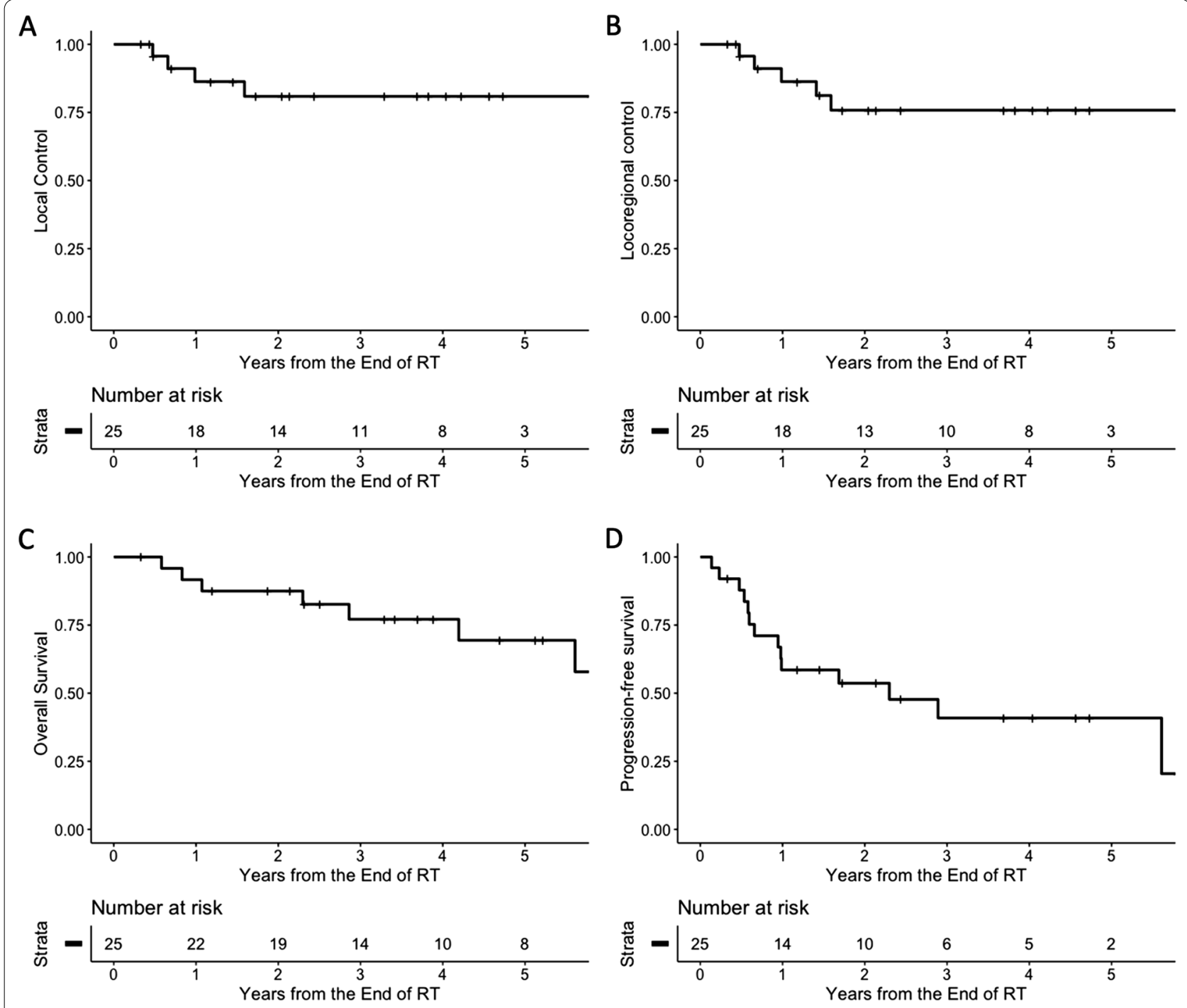

Fig. 2 Treatment outcomes. (a) Local control, (b) locoregional control, (c) overall survival, and (d) progression-free survival of the included patients

Table 3 Toxicity profile

\begin{tabular}{|c|c|c|c|c|c|}
\hline \multirow[t]{2}{*}{ Grade } & \multicolumn{5}{|l|}{ Type of toxicity } \\
\hline & $\begin{array}{l}\text { Urinary symptoms (other than } \\
\text { hematuria) }\end{array}$ & Hematuria & $\begin{array}{l}\text { Proctitis (other than } \\
\text { hematochezia) }\end{array}$ & Hematochezia & Others \\
\hline None & $11(52.4 \%)$ & $13(61.5 \%)$ & 13 (61.9\%) & $8(38.1 \%)$ & 20 (95.2\%) \\
\hline 1 & $3(14.3 \%)$ & $2(9.5 \%)$ & $6(28.6 \%)$ & $5(23.8 \%)$ & $0(0.0 \%)$ \\
\hline 2 & $5(23.8 \%)$ & $3(14.3 \%)$ & $2(9.5 \%)$ & $5(23.8 \%)$ & $1(4.8 \%)$ \\
\hline 3 & $2(9.5 \%)$ & $3(14.3 \%)$ & $0(0.0 \%)$ & $3(14.3 \%)$ & $0(0.0 \%)$ \\
\hline
\end{tabular}

In the univariate analysis, cumulative $D_{2 c c}$ of rectum was marginally correlated with occurrence of any grade of hematochezia $(P=0.051)$. No correlation was found with occurrence of grade $\geq 2(P=0.213)$ or $\geq 3$ hematochezia $(P=0.127)$. Cumulatve $D_{2 \mathrm{cc}}$ of bladder was not correlated with hematuria regardless of cutoff grade (any grade, $P=0.829 ;$ grade $\geq 2, P=0.318 ;$ grade $\geq 3$, $P=0.739)$. In the ROC curve analysis, cumulative $D_{2 \text { cc }}$ 

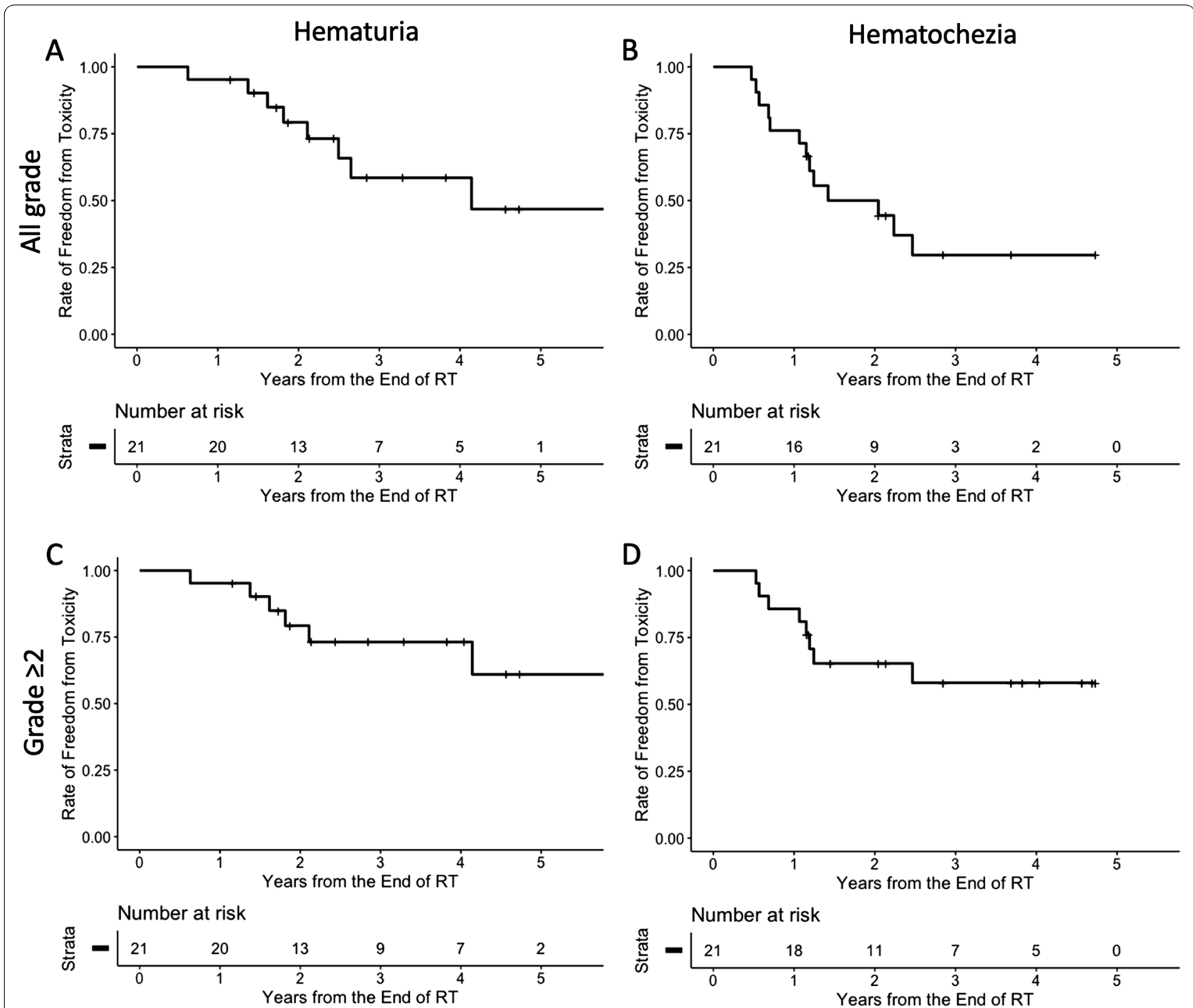

Fig. 3 Rate of freedom from toxicity. Rate of freedom from (a) hematuria with any grade, (b) hematochezia with any grade, (c) hematuria with grade 2 or worse, (d) hematochezia with grade 2 or worse

of rectum showed statistical significance for any grade of hematochezia (area under the curve [AUC] 0.7500, 95\% confidence interval [CI] 0.5281-0.9719), and the optimal threshold was 81.2 Gy EQD2 with a sensitivity of $61.5 \%$ and specificity of $100 \%$. The ROC curve is illustrated in Fig. 4.

\section{Discussion}

The present study evaluated SABR boost in patients who could not undergo brachytherapy, and reported four (16.0\%) local failures with a 3-year local control rate of $80.9 \%$. The reported local failure rate was comparable to a landmark study that employed concurrent chemoradiation and brachytherapy for locally advanced cervical cancer [8], but lower than recent studies that utilized MRI-based brachytherapy [9]. Although OS and PFS rates were relatively low in the current study, it should be noted that the enrolled patients in the present study were old and morbid. For instance, more than half of the patients from GOG 120 trial had age less than or equal to 50 years, and over $90 \%$ of the patients had Karnofsky performance status more than or equal to 70 [8]. Patients from RetroEMBRACE trial had median age of 53 [9]. In the present study, median age of the patients was 73.7 years, and $20 \%$ of the patients had ECOG performance score $2-3$. Toxicities were manageable in the majority of the patients, with no grade 4 or 5 adverse events. 


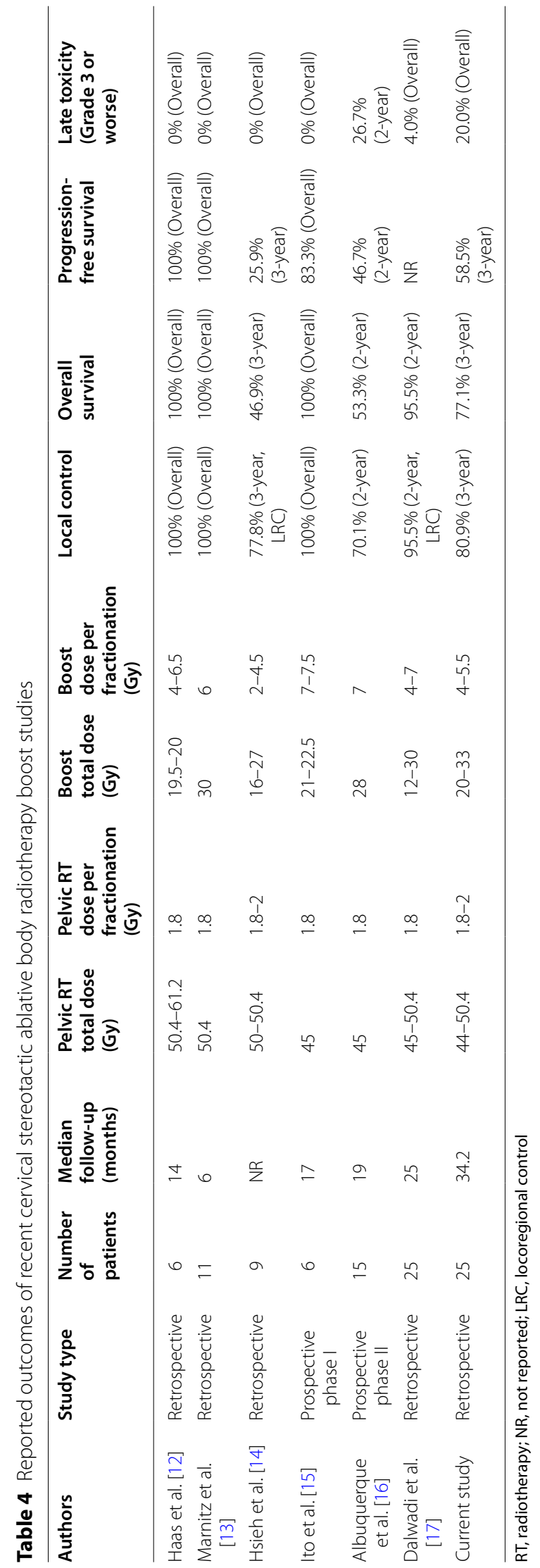




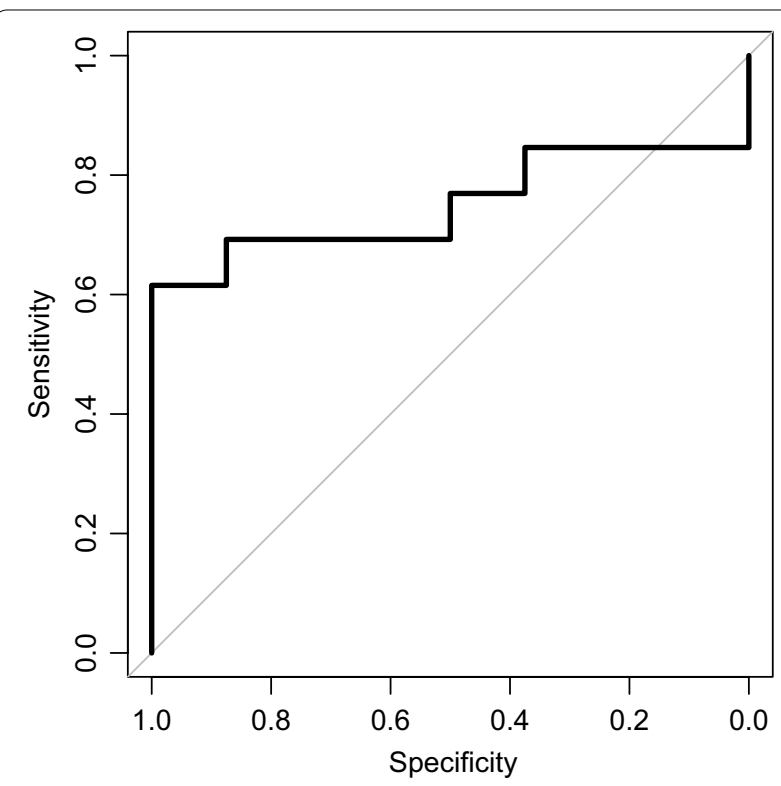

Fig. 4 Receiver operating characteristic curve for occurrence of hematochezia. Receiver operating characteristic curve for occurrence of any grade of hematochezia by rectal cumulative $D_{2 c c}$ EQD2

There have been several attempts to substitute brachytherapy boost with EBRT boost even before IMRT and SABR techniques became common. Although previous EBRT boost studies utilizing the 3D-CRT technique reported acceptable treatment outcomes and toxicity profiles $[10,11]$, the dose delivered to the tumor was often lower than brachytherapy boost or EBRT boost with modern techniques. For instance, in a study by Barraclough et al., a total dose of 60-65 Gy with conventional fractionation was delivered to $71 \%$ of the patients [3], which is lower than the median EQD2 of 75.5 Gy in the present study. With the adoption of stereotactic techniques as well as the advancements in imaging techniques during RT, several groups have reported treatment outcomes of cervical SABR boost after pelvic RT for locally advanced cervical cancer. The reported outcomes of recent SABR boost studies are summarized in Table 4. Along with the present study, these studies revealed an acceptable local control rate of 70 to $100 \%$, indicating that SABR boost may be sufficiently effective for cervical disease control, although a wide range of OS and PFS rates have been reported depending on the baseline patient status. It should be noted that patients who undergo cervical SABR boost tend to be older and have more comorbidities than other locally advanced cervical cancer patients who require brachytherapy boost [18].

The major concern of cervical SABR boost is the potentially high toxicity due to a higher dose to organs at risk (OARs). Brachytherapy has advantages over EBRT in the dose distribution to OARs, and previous RT plan studies that tried to mimic the dose distribution of brachytherapy by IMRT and proton beam demonstrated that EBRT plans were inferior to brachtherapy plans [19]. Although several studies did not report severe late toxicities [12, 13], these studies usually had a short follow-up period with a small number of patients, making them less representative. A recent prospective phase II study reported a 2 -year cumulative grade 3 or worse toxicity rate of $26.7 \%$, which was predominantly rectal, and two of the patients had grade 5 fistula formation [16]. The study used a dose-fractionation scheme of 28 Gy with 4 fractionations, equivalent to a BED of 47.6 Gy with an $\alpha / \beta$ ratio of 10 , which is relatively high. The current study had a median SABR boost BED of 37.5 Gy with an $\alpha / \beta$ ratio of 10 , and although manageable, $20 \%$ of the patients experienced grade 3 toxicity. A recent prospective phase I study showed that a dose-fractionation scheme of 22.5 Gy in 3 fractions was tolerable, which is equivalent to a BED of 39.4 Gy with an $\alpha / \beta$ ratio of 10 [15]. Additional studies are needed to determine a safe and effective dose-fractionation scheme.

In the univariate analysis and ROC curve analysis of the current study, we demonstrated that cumulative $\mathrm{D}_{2 \mathrm{cc}}$ EQD2 of rectum was marginally correlated with the occurrence of hematochezia as a late toxicity. It can be easily assumed that the anterior rectal wall would be irradiated with high dose in both pelvic RT and SABR boost. Thus, it can be concluded from the findings of the present study that limiting the dose to the anterior rectal wall might be helpful in reducing the rectal toxicity of cervical SABR boost. The dosimetric analysis of the previously mentioned prospective phase II study showed that the percentage of the rectal circumference receiving 15 Gy was associated with the development of rectovaginal fistula [16]. In case of brachytherapy, guidelines from the American Brachytherapy Society suggest a rectal dose limit of $\mathrm{D}_{2 \mathrm{cc}} 75$ Gy EQD2 for brachytherapy with $3 \mathrm{D}$ imaging [20]. The exact thresholds vary, but limiting the dose to some portion of the anterior rectal wall is a common feature. In contrast, for the occurrence of hematuria, cumulative $\mathrm{D}_{2 \mathrm{cc}}$ EQD2 of bladder showed no statistical significance in the present study. A prospective study with planned dosimetric analysis and precise assessment of adverse events is needed to determine useful OAR dose thresholds.

The present study has several limitations. Considering the small number of enrolled patients, the cohort of this study may not be representative enough to address the feasibility of cervical SABR boost. Additionally, the patients were older and more morbid compared to previous landmark studies, and this may have affected the assessment of the local control rate. Toxicity may also 
have been underreported due to the retrospective nature of this study. However, the present study has a relatively larger patient cohort and longer follow-up period than previous studies. Considering that cervical SABR boost is utilized secondary to brachytherapy, recruiting a large number of patients may be very challenging; consequently, the results can be considered meaningful even with this size of the cohort. Furthermore, as there is a higher chance of brachytherapy being considered not feasible in old and morbid patients, this study shows possible outcomes of patient groups in the real world.

\section{Conclusion}

SABR boost for patients with locally advanced cervical cancer who are unable to undergo brachytherapy was found to be effective with manageable toxicity. Although it cannot substitute brachytherapy, SABR boost can be a treatment option when brachytherapy is not possible. The exact dose-fractionation scheme and dose limitation for OARs need to be addressed in further studies to ensure the safety of cervical SABR boost.

\section{Acknowledgements}

Not applicable.

\section{Authors' contributions}

THL was responsible for data analysis and manuscript writing. YBK and JSK was responsible for project administration. CS, KK, JHN, KHS and JBC contributed to data collection. IAK and KYE contributed to study designing and project administration. All authors contributed to the revision of the manuscript and approval of the final manuscript.

\section{Funding}

Not applicable.

\section{Availability of data and materials}

The datasets used and/or analysed during the current study are available from the corresponding author on reasonable request.

\section{Declarations}

\section{Ethics approval and consent to participate}

This study was approved by the Institutional Review Board of Seoul National University Bundang Hospital (IRB Number B-2004/606-110) before collecting the patient information. Informed consent was waived.

\section{Consent for publication}

Not applicable.

\section{Competing interests}

The authors declare that they have no competing interests.

\section{Author details}

${ }^{1}$ Department of Radiation Oncology, Seoul National University Hospital, Seoul, Republic of Korea. ${ }^{2}$ Department of Radiation Oncology, Seoul National University Bundang Hospital, 82 Gumi-ro 173 beon-gil, Bundang-gu, Seongnam 13620, Republic of Korea. ${ }^{3}$ Department of Obstetrics and Gynecology, Seoul National University Bundang Hospital, Seongnam, Republic of Korea.

Received: 7 May 2021 Accepted: 4 August 2021

Published online: 12 August 2021

\section{References}

1. Han K, Milosevic M, Fyles A, Pintilie M, Viswanathan AN. Trends in the utilization of brachytherapy in cervical cancer in the United States. Int J Radiat Oncol Biol Phys. 2013;87(1):111-9.

2. Viswanathan AN, Moughan J, Small W, Levenback C, Iyer R, Hymes S, et al. The quality of cervical cancer brachytherapy implantation and the impact on local recurrence and disease-free survival in Radiation Therapy Oncology Group prospective trials 0116 and 0128. Int J Gynecol Cancer. 2012;22(1):123-31.

3. Barraclough LH, Swindell R, Livsey JE, Hunter RD, Davidson SE. External beam boost for cancer of the cervix uteri when intracavitary therapy cannot be performed. Int J Radiat Oncol Biol Phys. 2008;71(3):772-8.

4. Kim H, Kim YS, Joo JH, Eom KY, Park W, Kim JH, et al. Tumor boost using external beam radiation in cervical cancer patients unable to receive intracavitary brachytherapy: outcome from a multicenter retrospective study (Korean Radiation Oncology Group 1419). Int J Gynecol Cancer. 2018;28(2):371-8.

5. Mahmoud O, Kilic S, Khan AJ, Beriwal S, Small W. External beam techniques to boost cervical cancer when brachytherapy is not an optiontheories and applications. Ann Transl Med. 2017;5(10):207.

6. Lim K, Small W, Portelance L, Creutzberg C, Jürgenliemk-Schulz IM, Mundt A, et al. Consensus guidelines for delineation of clinical target volume for intensity-modulated pelvic radiotherapy for the definitive treatment of cervix cancer. Int J Radiat Oncol Biol Phys. 2011;79(2):348-55.

7. Pötter R, Haie-Meder C, Van Limbergen E, Barillot I, De Brabandere M, Dimopoulos J, et al. Recommendations from gynaecological (GYN) GEC ESTRO working group (II): concepts and terms in 3D image-based treatment planning in cervix cancer brachytherapy -3D dose volume parameters and aspects of 3D image-based anatomy, radiation physics, radiobiology. Radiother Oncol. 2006;78(1):67-77.

8. Rose PG, Bundy BN, Watkins EB, Thigpen JT, Deppe G, Maiman MA, et al. Concurrent Cisplatin-based radiotherapy and chemotherapy for locally advanced cervical cancer. N Engl J Med. 1999;340(15):1144-53.

9. Sturdza A, Pötter R, Fokdal LU, Haire-Meder C, Tan LT, Mazeron R, et al. Image guided brachytherapy in locally advanced cervical cancer: Improved pelvic control and survival in RetroEMBRACE, a multicenter cohort study. Radiother Oncol. 2016;120(3):428-33.

10. Chan P, Yeo I, Perkins G, Fyles A, Milosevic M. Dosimetric comparison of intensity-modulated, conformal, and four-field pelvic radiotherapy boost plans for gynecologic cancer: a retrospective planning study. Radiat Oncol. 2006;1(1):1-10.

11. Matsuura K, Tanimoto H, Fujita K, Hashimoto Y, Murakami Y, Kenjo M, et al. Early clinical outcomes of 3D-conformal radiotherapy using accelerated hyperfractionation without intracavitary brachytherapy for cervical cancer. Gynecol Oncol. 2007;104(1):11-4.

12. Haas JA, Witten MR, Clancey O, Episcopia K, Accordino D, Chalas E. CyberKnife boost for patients with cervical cancer unable to undergo brachytherapy. Front Oncol. 2012;2(MAR):1-5. 
13. Marnitz S, Köhler C, Budach V, Neumann O, Kluge A, Wlodarczyk W, et al. Brachytherapy emulating robotic radiosurgery in patients with cervical carcinoma. Radiat Oncol. 2013;8(1):2-9.

14. Hsieh CH, Tien HJ, Hsiao SM, Wei MC, Wu WY, Sun HD, et al. Stereotactic body radiation therapy via helical tomotherapy to replace brachytherapy for brachytherapy-unsuitable cervical cancer patients_-a preliminary result. Onco Targets Ther. 2013;6:59-66.

15. Ito K, Kito S, Nakajima Y, Shimizuguchi T, Ogawa H, Nihei K, et al. Determining the recommended dose of stereotactic body radiotherapy boost in patients with cervical cancer who are unsuitable for intracavitary brachytherapy: a phase I dose-escalation study. Jpn J Clin Oncol. 2019;49(9):856-61.

16. Albuquerque K, Tumati V, Lea J, Ahn C, Richardson D, Miller D, et al. A phase II trial of stereotactic ablative radiation therapy as a boost for locally advanced cervical cancer. Int J Radiat Oncol Biol Phys. 2020;106(3):464-71.

17. Dalwadi S, Echeverria A, Jhaveri P, Bui T, Waheed N, Tran D, et al. Noninvasive stereotactic ablative boost in patients with locally advanced cervical cancer. Int J Gynecol Cancer. 2020;30(11):1684-8.
18. O'Donnell B, Shiao JC, Pezzi TA, Waheed N, Sharma S, Bonnen MD, et al. Stereotactic body radiation therapy, intensity-modulated radiation therapy, and brachytherapy boost modalities in invasive cervical cancer: a study of the national cancer data base. Int J Gynecol Cancer. 2018;28(3):563-74.

19. Georg D, Kirisits C, Hillbrand M, Dimopoulos J, Pötter R. Image-guided radiotherapy for cervix cancer: high-tech external beam therapy versus high-tech brachytherapy. Int J Radiat Oncol Biol Phys. 2008;71(4):1272-8.

20. Viswanathan AN, Beriwal S, De Los Santos JF, Demanes DJ, Gaffney D, Hansen J, et al. American Brachytherapy Society consensus guidelines for locally advanced carcinoma of the cervix. Part II: High-dose-rate brachytherapy. Brachytherapy. 2012;11(1):47-52.

\section{Publisher's Note}

Springer Nature remains neutral with regard to jurisdictional claims in published maps and institutional affiliations.
Ready to submit your research? Choose BMC and benefit from:

- fast, convenient online submission

- thorough peer review by experienced researchers in your field

- rapid publication on acceptance

- support for research data, including large and complex data types

- gold Open Access which fosters wider collaboration and increased citations

- maximum visibility for your research: over $100 \mathrm{M}$ website views per year

At BMC, research is always in progress.

Learn more biomedcentral.com/submissions 